\title{
THE PROJECTION OF THE MIDLINE AND INTRALAMINAR NUCLEI OF THE THALAMUS OF THE RABBIT
}

\author{
BY \\ W. M. COWAN and T. P. S. POWELL \\ From the Department of Human Anatomy, University of Oxford
}

Electrophysiological studies have indicated that the nuclei of the midline and internal medullary lamina of the thalamus together constitute a functionally distinct system which may be of considerable importance in the maintenance and control of normal cerebral activity. Since most of the physiological observations on this system have recently been collated in the publication of the symposium on "Brain Mechanisms and Consciousness" (Council for International Organizations of Medical Sciences, 1954) a detailed review of the literature will not be given here. Suffice it to say that whereas each of the principal thalamic nuclei is related to a localized cortical area, stimulation studies have shown that the midline and intralaminar nuclei are capable of exerting widespread effects on the activity of the cerebral cortex. The normal pathways by which these effects are mediated are still obscure since it has long been known that these nuclei have no direct connexion with the neopallial cortex as they do not undergo retrograde cell degeneration even after decortication. That their projection is extrathalamic is, however, well established (Rose and Woolsey, 1943, 1949) and there is some evidence to suggest that they may be related to the corpus striatum (Stefens and Fortuyn, 1953 ; Powell and Cowan, 1954).

In view of the important functional significance attributed to these nuclei more precise information concerning their efferent connexions is desirable.

In the present work the projection of the individual elements of this group has been studied in the thalamus of the rabbit using the technique of retrograde cell degeneration after lesions in the rostral part of the cerebral hemisphere.

\section{Material and Methods}

Thirty-five rabbits of different ages were used in this study. In the earlier experiments lesions were placed in the basal forebrain areas by inserting through a trephine hole in the skull either a fine ophthalmic knife or an insulated electrode. In the later experiments the use of a stereotaxic instrument with a set of previously determined coordinates of the rabbit forebrain permitted more accurate placing of the lesions. The animals were allowed to survive for periods of one to three months after operation. The brains were removed and fixed in $70 \%$ alcohol and blocks containing the entire cerebral hemispheres were embedded in paraffin wax and sectioned coronally at 20 to $25 \mu$. Every fifth section was mounted serially and stained with methylene blue or thionine and every sixth section with activated protargol. In most of the experiments the lesions were confined to one side but in a few animals bilateral lesions were made, the appropriate hemisphere of the latter being indicated in the text by the suffix R. or L. A number of series of sections of normal brains stained with both thionine and protargol were available for comparison with the experimental material. Essentially four different types of lesion were placed. In the first series of experiments almost the entire rostral portion of the hemisphere in front of the thalamus was ablated. In the second group a variety of lesions were placed in the internal capsule and basal forebrain areas to determine the gross projection and pathway of the efferent fibres of the midline and intralaminar nuclei. In the last two groups an attempt was made to localize the projection of individual elements of this system by controlled ablations of the cortex on the medial surface of the hemisphere and by a series of overlapping lesions in the corpus striatum. In addition to representative experiments of these groups, a few experiments which serve to exclude some of the adjacent structures will be described.

In view of the different terminology which has been used by previous authors for the nuclei of the midline and intralaminar system a brief reference to the nomenclature used in this paper is necessary. With one exception it will be the same as that adopted in the previous study in the rat (Powell and Cowan, 1954); in accordance with the description of Gurdjian (1927) the cell mass immediately adjacent to the mamillo-thalamic tract was called the medio-ventral nucleus but in the present study on the rabbit we have followed Rose and Mountcastle (1952) in calling this the ventro-medial nucleus. On the other hand the term nucleus reuniens has been retained for the most ventral nucleus of the midline group which Rose (Rose and Woolsey, 1948 ; Rose and Mountcastle, 1952) terms the medio-ventral nucleus; this same nucleus has been subdivided by Fortuyn (1950) into a paramedian and a submedial nucleus. The rhomboid nucleus is the name given to the group of deeply staining cells immediately dorsal to the nucleus 


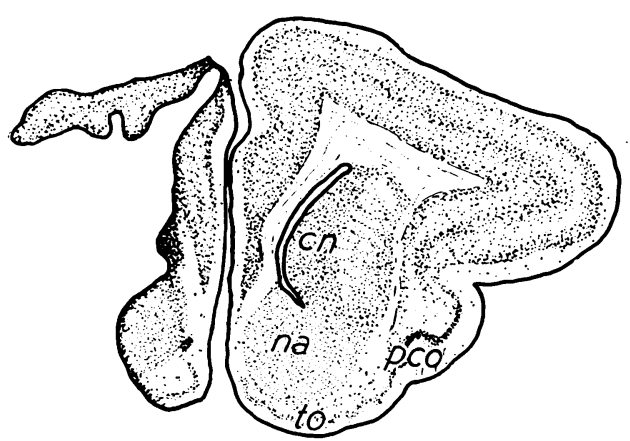

F F. 1.-Site and extent of the lesion in experiment $\mathbf{R} 2$.

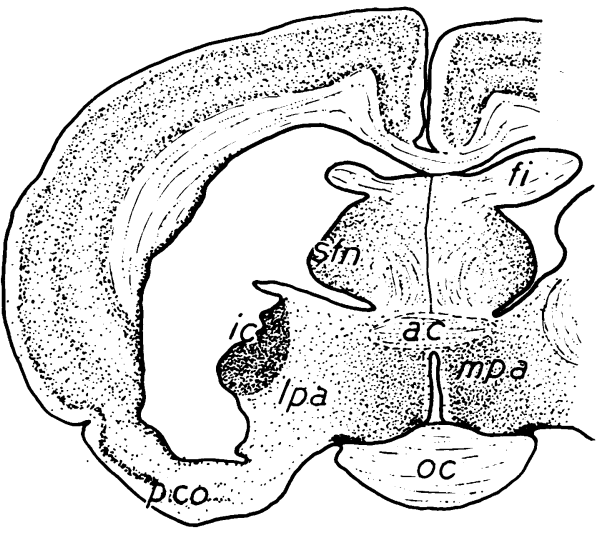

$$
\begin{aligned}
& \text { s f } \mathbf{n}=\text { septofimbrial nucleus } \\
& \text { i c }=\text { internal capsule } \\
& \text { l p a }=\text { lateral preoptic area } \\
& \text { p c o }=\text { pyriform cortex } \\
& \text { a c }=\text { anterior commissure } \\
& \text { o c }=\text { optic chiasma } \\
& \text { f i }=\text { fimbria } \\
& \text { m p a }=\text { medial preoptic area }
\end{aligned}
$$

olfactory tubercle

reuniens, the term $n$. centralis medialis referring to the midline cell mass between the two paracentral nuclei (cf. Rose and Woolsey, 1943). Since it is not possible to distinguish clearly between the parafascicular and centro-median nuclei either in normal or in the experimental material, these two elements will be described together simply as the parafascicular nucleus.

\section{Results}

In Rabbit 2, which is representative of the first group of experiments, the cerebral hemisphere was completely ablated in front of the genu of the corpus callosum. Examination of the serial sections shows that behind this level the lesion is somewhat less extensive, but with the exception of the neocortex on the dorso-lateral aspect of the hemisphere all the structures lateral to the anterior horn of the lateral ventricle have been destroyed, including the entire striatum, the anterior limb of the internal capsule, the lateral preoptic area, and the anterior third of the amygdaloid complex. The septum, i.e., the medial and lateral septal nuclei, the vertical limb of the diagonal band nucleus, and the anterior hippocampal cortex are intact. The medial preoptic and anterior hypothalamic areas have also escaped damage, since the lesion narrows considerably in its posterior part and is here confined to the body of the caudate nucleus and the immediately adjacent part of the internal capsule (Fig. 1). The retrograde cell degeneration in the thalamus is extensive and involves to a varying degree all the principal nuclei and most of the midline and intralaminar group (Fig. 2). Of the latter the nuclei reuniens, the rhomboideus, centralis medialis, paracentralis, cen- tralis lateralis, and parafascicularis have undergone complete atrophy. The parataenial nucleus shows a severe cell loss throughout its antero-posterior extent and especially in the ventro-lateral twothirds of its cross sectional area (Plate 1). The ventral vertical portion of the anterior paraventricular nucleus shows a definite loss of cells with shrinkage of the remaining cells but there is no appreciable change in the posterior paraventricular nucleus. In the anterior part of the reticular nucleus* there is a marked gliosis together with severe shrinkage and compacting of the cells and possibly some cell loss; posteriorly this nucleus shows little or no change. Of particular significance in the main thalamic nuclei is the complete retrograde degeneration of the nucleus ventralis anterior and the ventro-medial nucleus. With the exception of the antero-dorsal, the lateral and pretectal nuclei, and the medial and lateral geniculate nuclei, in all of which a small number of normal cells persist, the other principal nuclei are completely degenerate.

This and the other experiments in the group confirm the findings of Rose and Woolsey (1943) and our previous observations in the rat (Powell and Cowan, 1954) that the midline and intralaminar nuclei will degenerate following large lesions in the rostral telencephalon. The next group of experiments suggests that these nuclei project to the striatum and the adjacent neocortex and in their projection are largely independent of the principal

\footnotetext{
* As here used, the term reticular nucleus refers to the collection of cells in the external medullary lamina of the thalamus and does not refer to the brain-stem reticular formation.
} 


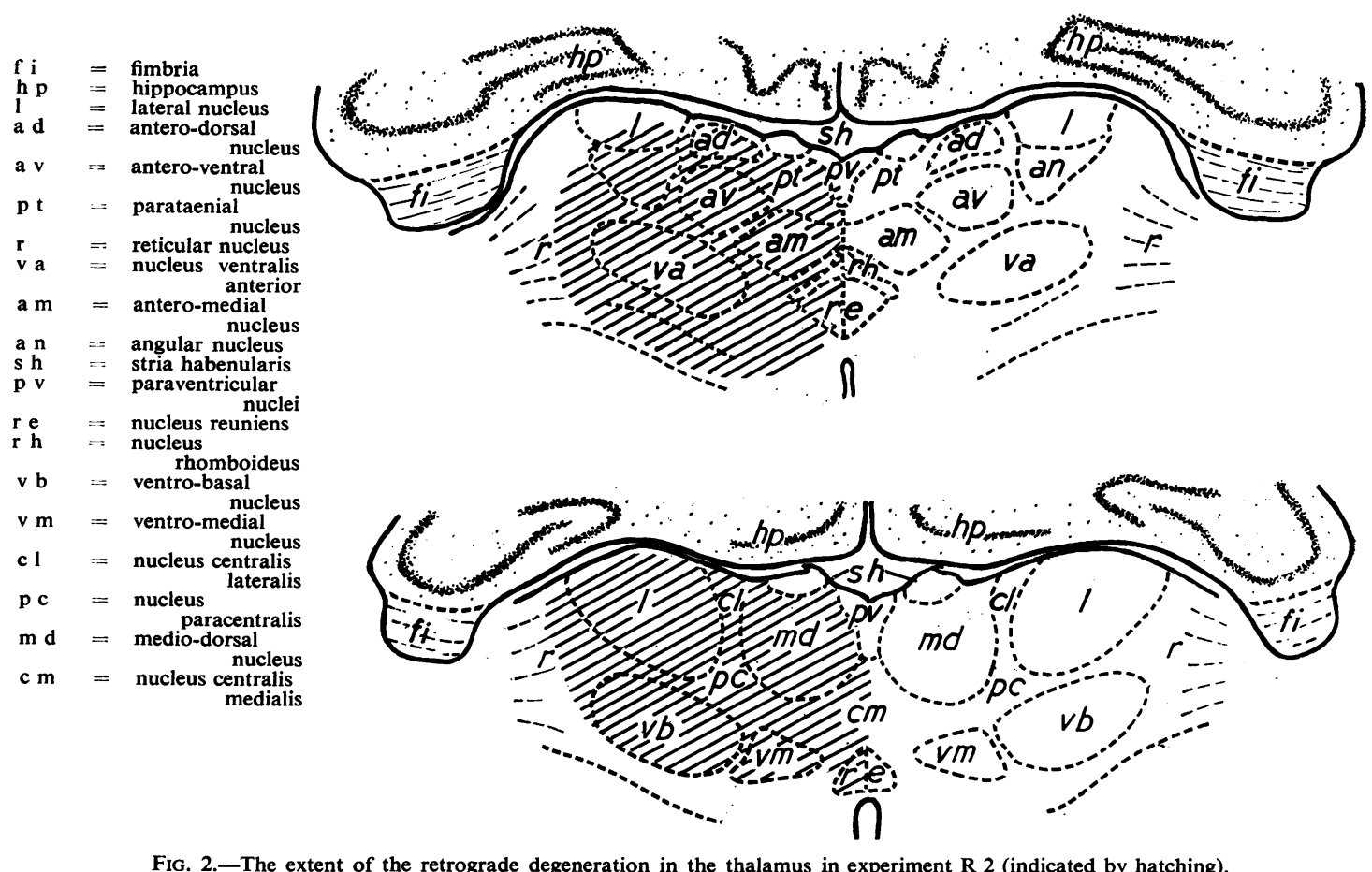

Fig. 2.-The extent of the retrograde degeneration in the thalamus in experiment $R 2$ (indicated by hatching).

nuclei; it also indicates the pathway of their projection fibres.

Of the second group of experiments only Rabbits 15 and 60 will be described. In Rabbit 15 the lesion was confined to structures abutting on the medial surface of the hemisphere as far ventrally as the olfactory tubercle. In front of the level of the septum the caudate nucleus, the ventral half of the internal capsule, the putamen, and the nucleus accumbens have been completely destroyed; the rostral two-thirds of the septum of this side have also been ablated, but posteriorly the damage to the striatum becomes restricted to the medial half of the caudate nucleus and at the level of the posterior third of the septum it is minimal. The main portion of the lesion then extends back into the lateral preoptic area and the lateral half of the medial preoptic area destroying the anterior commissure and the ventromedial quarter of the internal capsule at this level. The striatum (putamen and caudate nucleus) adjacent to the damaged internal capsule has suffered only marginal involvement. The lesion stops abruptly at the level of the anterior margin of the optic chiasma, the hypothalamus being unaffected.

In the thalamus the degeneration is limited largely to the nuclei of the midline and the internal medullary lamina. The nuclei reuniens, rhomboideus, centralis medialis, paracentralis, centralis lateralis and the parataenial show a marked cell loss throughout their antero-posterior extent. In the parafascicular nucleus there is a moderate degree of cell loss in its antero-medial part, but the rest of the nucleus is unchanged. The ventral vertical parts of the anterior and posterior paraventricular nuclei are also severely degenerate. Slight cell loss and cell shrinkage are seen in the medial thirds of the nucleus ventralis anterior and the reticular nucleus, accompanied by an intense gliosis which extends into the inferior thalamic radiation. Of the other principal nuclei, only the antero-medial, medio-dorsal, ventro-medial nuclei and the medial halves of the antero-dorsal, anteroventral, ventro-basal and ventro-lateral nuclei show any retrograde cell degeneration. The thalamic degeneration in this experiment is obviously the result of the interruption of fibres in the internal capsule, again confirming our previous observation (Powell and Cowan, 1954) that the efferent fibres of these nuclei traverse the lower medial portion of the internal capsule.

In Rabbit 60 a well circumscribed electrolytic lesion was placed in the rostral part of the hemisphere destroying most of the head of the caudate 

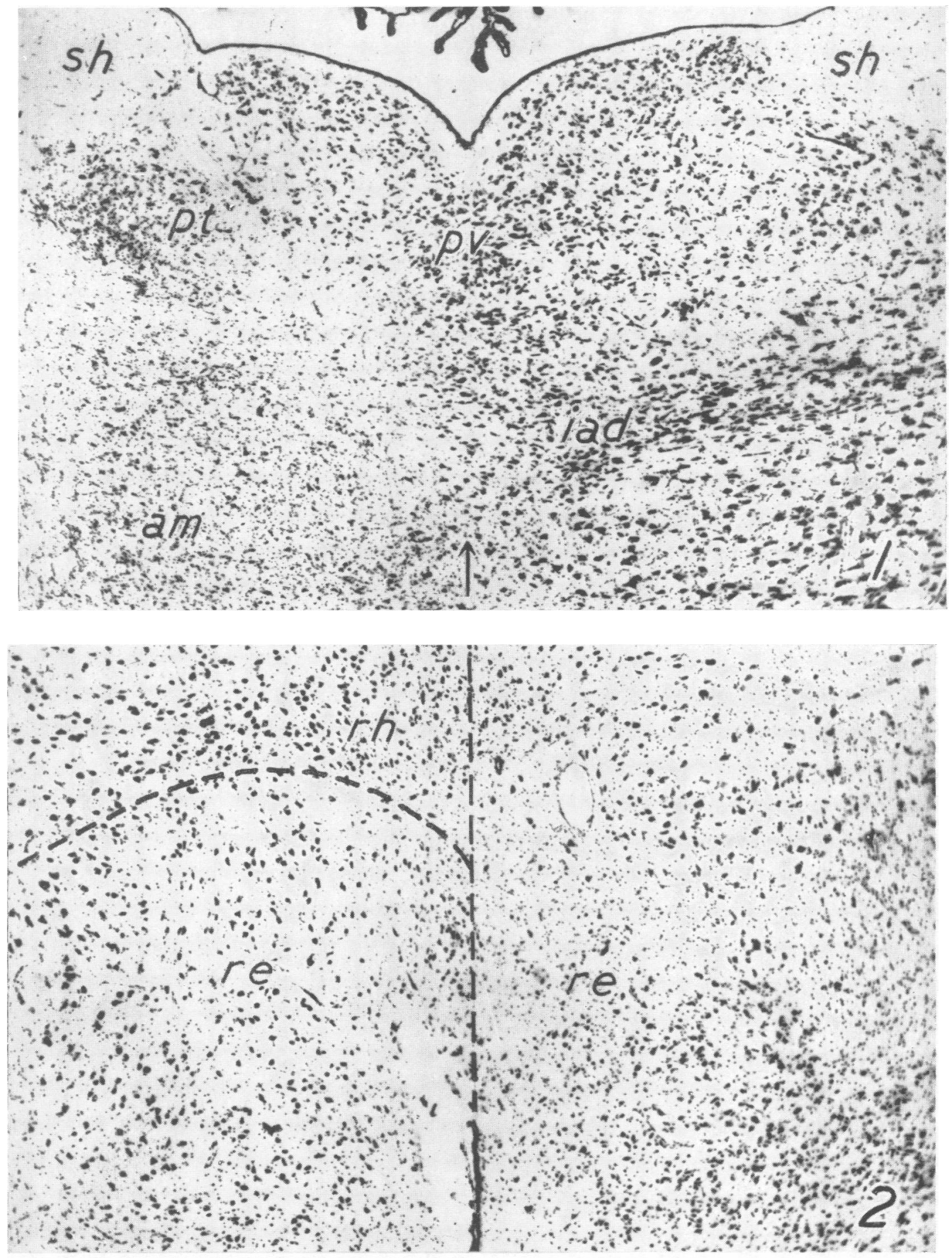

The sections were stained with either methylene blue or thionine. The magnification of all photomicrographs is 62.

Plate 1.--Photomicrograph showing retrograde cell degeneration in the parataenial, ventral part of the paraventricular, interantero-dorsal, and antero-medial nuclei in experiment $\mathbf{R} 2$.

Plate 2.-Degeneration in the nuclei reuniens and rhomboideus in experiment $R 54$. 

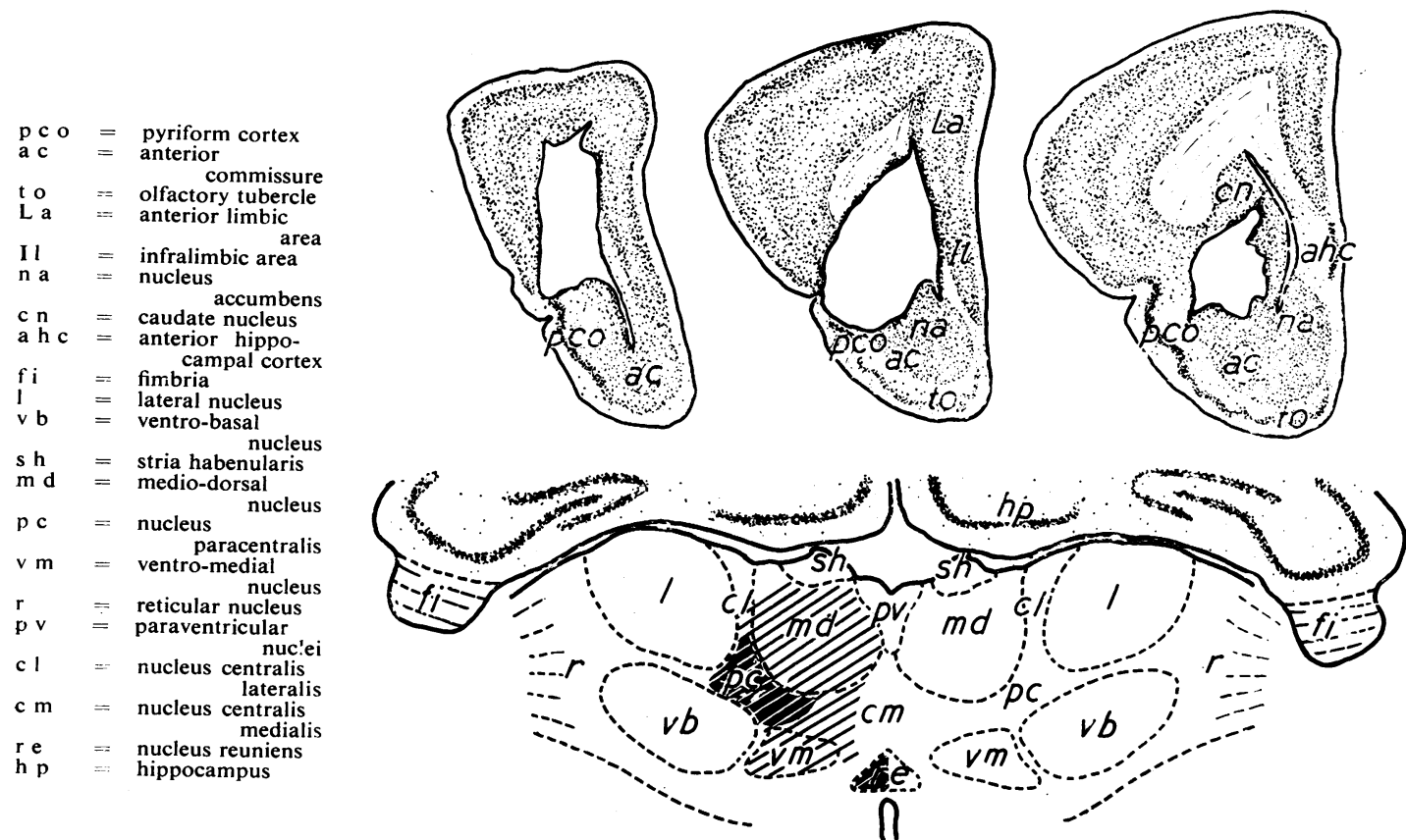

FIG. 3.-Site and extent of lesion in experiment $R 60$ together with the thalamic degeneration in this experiment. There is almost total degeneration in nn. paracentralis and reuniens while there is severe cell loss in nn. centralis lateralis, medio-dorsalis, and ventro-medialis. nucleus and the adjacent centrum ovale. In its antero-posterior length the lesion extends from the level of fusion of the anterior olfactory nucleus with the overlying neocortex back to just behind the genu of the corpus callosum. Rostral to the genu the head of the caudate nucleus, the putamen, and the dorsal margin of the nucleus accumbens have been completely destroyed. Immediately behind this. level the lesion is restricted to the lateral part of the caudate nucleus and the adjacent middle portion of the internal capsule (Fig. 3). The resulting retrograde cell degeneration is found mainly in the midline and intralaminar group of thalamic nuclei (Fig. 3). The nucleus reuniens and the rhomboid nucleus have undergone severe cell loss, the remaining cells being shrunken and pyknotic. The nucleus centralis medialis shows a partial cell loss which is most severe in its lateral half where it adjoins the almost totally degenerate paracentral nucleus. The nucleus centralis lateralis is likewise severely degenerate in its medial half, but laterally there is no change apart from a slight gliosis. In the rostral part of the parafascicular nucleus where it adjoins the medio-dorsal nucleus there are slight changes in the form of shrinkage and compacting of the cells, together with a moderate degree of gliosis. The remaining midline nuclei (the para- taenial and the anterior and posterior para- $\delta$ ventricular) show no change. Shrinking and compacting of the constituent cells together witho severe gliosis are present in the adjoining medial portions of the nucleus ventralis anterior and the reticular nucleus. The other principal nuclei which show changes are the antero-medial, dorso-medial, and ventro-medial, in all of which there is a severe diffuse cell loss and gliosis. The course of the degenerated fibres is indicated by a band of dense gliosis in the ventral third of the internal capsule.

In the subsequent two groups of experiments an attempt was made to determine to what extent the cortical and subcortical structures, which were damaged in the previous experiments, are implicated in the projection of the midline and intralaminar nuclei. In the first of these groups the lesions were strictly limited to the cortex on the medial surface of the hemisphere involving principally the anterior limbic and infralimbic areas. Some of these lesions were placed primarily for a study of the relationships between the cingulate cortex and the mamillary nuclei (Cowan and Powell, 1954), and as the projection of the anterior nuclei of the thalamus to the different areas of this cortex has already been established by Rose and Woolsey (1948) the degeneration in these nuclei will not be described in detail. 

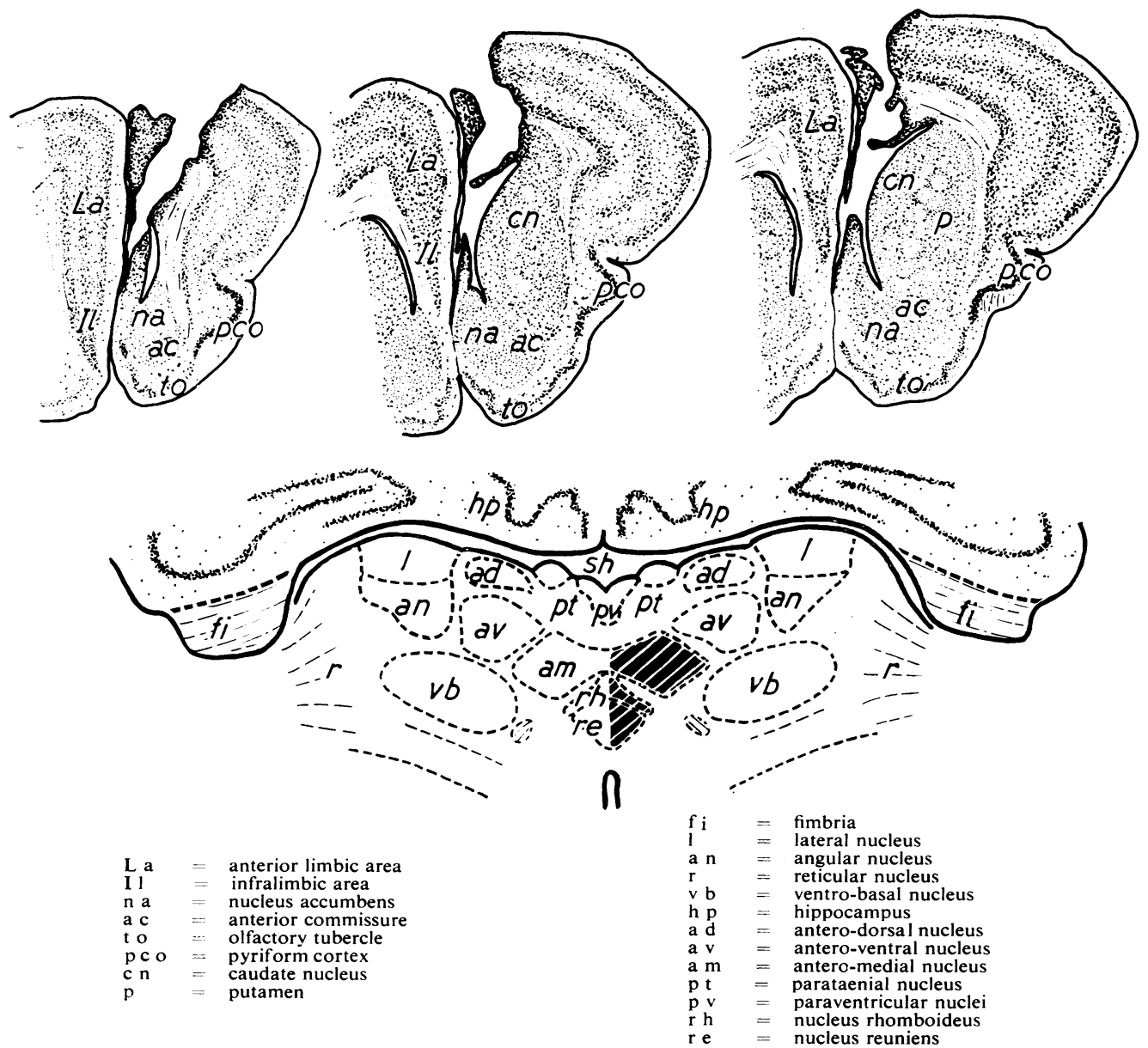

FIG. 4.-Extent of the lesion and the resulting thalamic degeneration in experiment $R 54$

In Rabbit 54 the entire neocortex of the medial surface of the hemisphere was ablated from the posterior margin of the orbito-frontal cortex back to the genu of the corpus callosum. The serial sections show that the whole of the anterior limbic and infralimbic areas and the prefrontal agranular cortex on the medial surface have been destroyed together with the rostral part of the lateral septal nucleus. It should be emphasized, however, that at no point does the lesion encroach on the nucleus accumbens or the caudate nucleus (Fig. 4).

The retrograde cell degeneration in this experiment is confined to the nuclei reuniens and the rhomboideus of the midline group while the only principal nuclei which show cell loss are the antero-medial and the medial third of the ventro-lateral (Plate 2). The absence of degeneration in the parataenial nucleus and in the intralaminar group should be particularly noted (Fig. 4).
The extent of the cortical damage in four similar experiments of this group is shown in Fig. 5. In the thalamus of these hemispheres cell loss has occurred in the midline nuclei reuniens and rhomboideus and also in the various elements of the anterior and ventral principal nuclei.

The cortical damage in experiment $R 35 R$ is mainly in the anterior limbic area, but in addition the postero-dorsal margin of the infralimbic field just above and in front of the genu of the corpus callosum and the precentral agranular areas are involved (Fig. 5). The fibres to the posterior cingulate and retro-splenial areas have also been interrupted. Of the midline thalamic nuclei, degeneration is found only in the nucleus reuniens in which slight cell loss is present throughout most of the cross-sectional area but is more marked in the dorsal third ; similar changes are found in the three anterior nuclei and the ventral nuclei. 
In all of the above experiments the anterior nuclei have degenerated in addition to the nucleus reuniens and the rhomboid nucleus. That the latter two have an independent projection, however, is shown in $\mathrm{R} 24 \mathrm{~L}$, in which the cortical damage is confined to the posterior two-thirds of the infralimbic area (Fig. 5) with a marginal involvement of the nucleus accumbens and the lateral septal nucleus. There is a diffuse cell loss throughout the antero-posterior extent of the nucleus reuniens and slight cell loss in the rhomboid nucleus. On the other hand there is no change in any of the anterior nuclei or the medio-dorsal nucleus.
In $\mathrm{R} 55 \mathrm{R}$ the caudate nucleus and the nucleus accumbens have been slightly involved in addition to most of the cortex on the medial surface. At the rostral end of the striatum there is a narrow knife cut into the dorso-lateral margin of the nucleus accumbens and this extends back to involve the medial and ventral parts of the caudate nucleus and the adjacent ventralmost part of the internal capsule. The only difference in the distribution of the thalamic degeneration in this hemisphere as compared with the experiments of the previous group is the diffuse cell loss throughout the entire anteroposterior extent of the parataenial nucleus.

FIG. 5.- The extent of the cortical lesions in experiments $R 55 \mathrm{~L}, \mathrm{R} 10, \mathrm{R} 27$ ( $\mathrm{R}$ and L), $\mathrm{R} 24 \mathrm{~L}$, and $\mathrm{R} 35 \mathrm{R}$ (sub-divisions of limbic cortex after Rose and Woolsey, 1948).
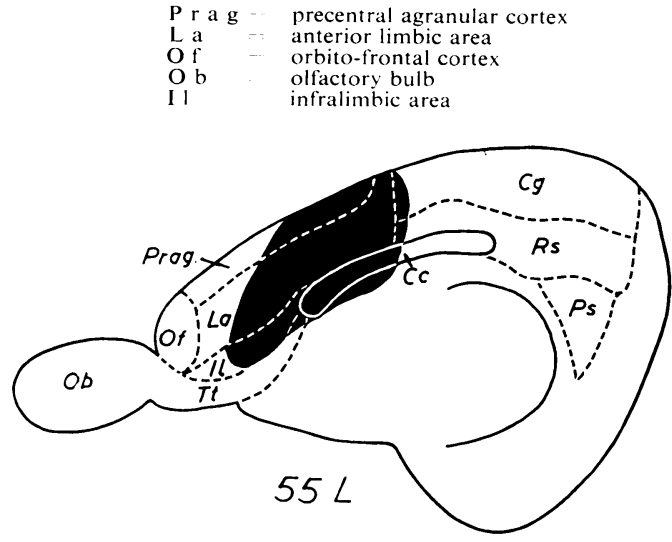

$55 \angle$
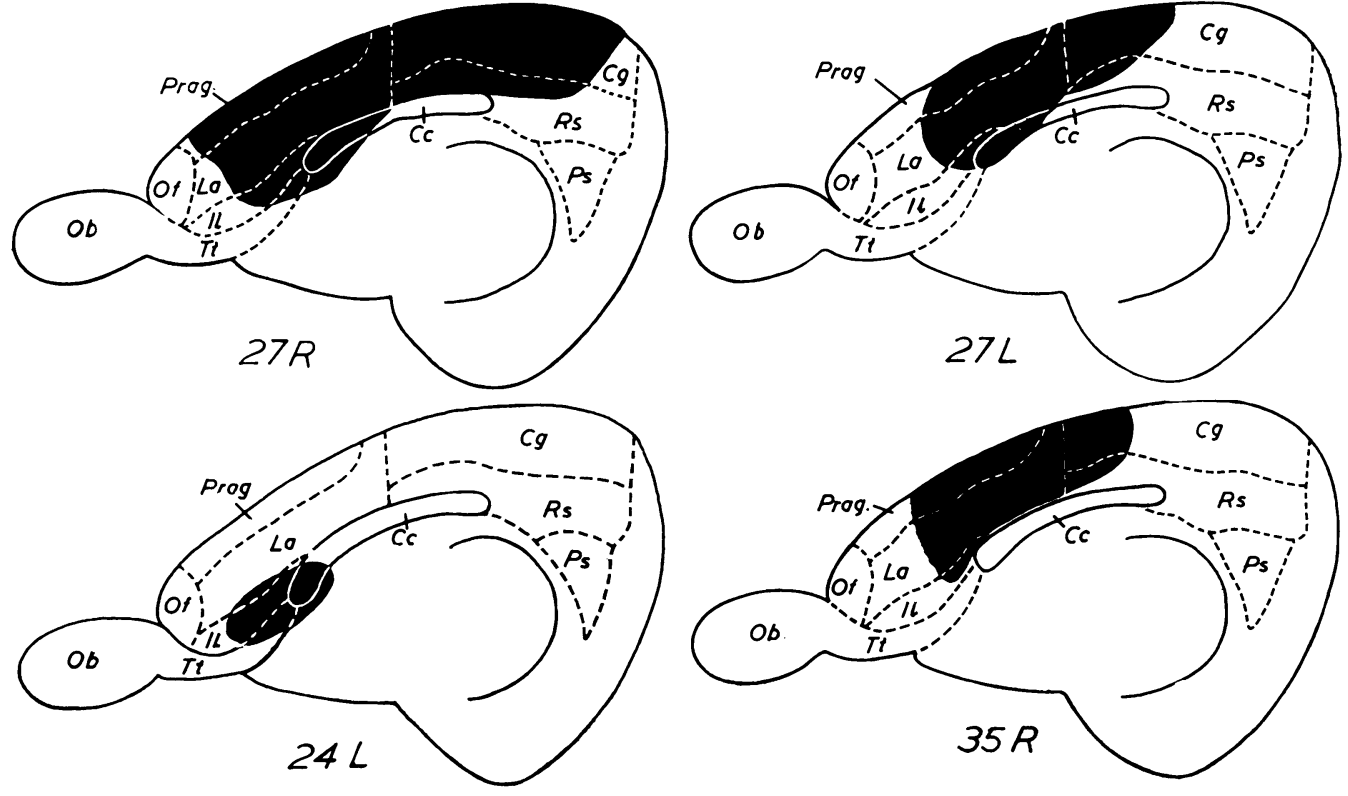

T t taenia tecta

C. $\mathrm{g}$ cingulate area (posterior cingulate cortex)

$\mathrm{R} s \quad$ retro-splenial area

C c corpus callosum
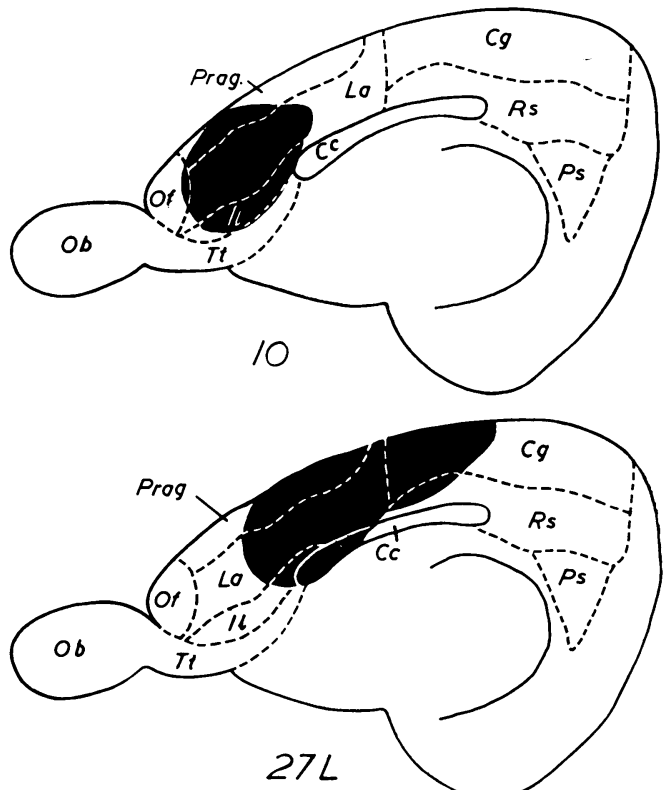

$27 L$

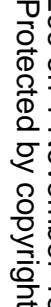

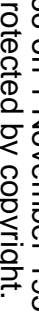




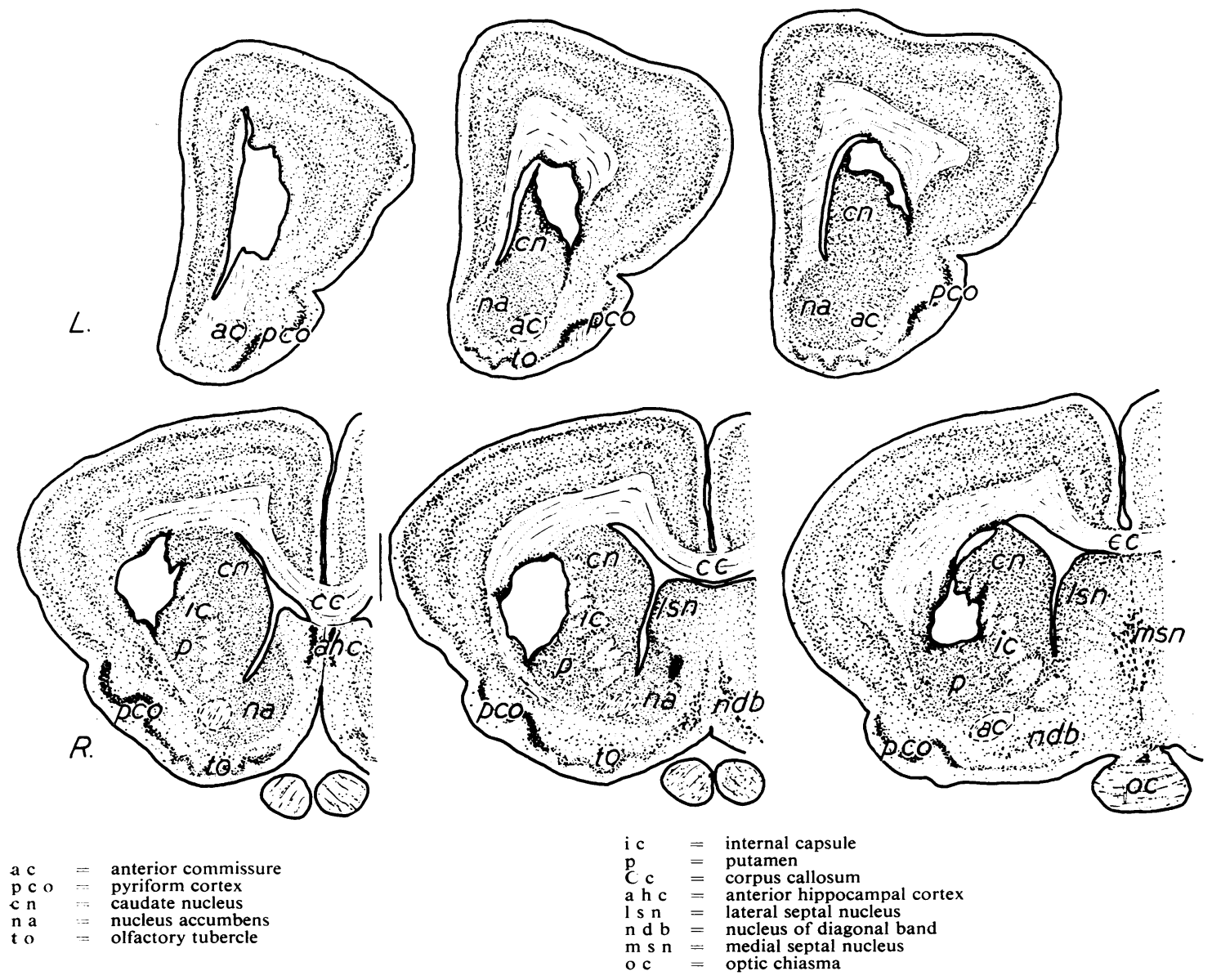

FiG. 6.-Site of the lesion in experiment R $251 \mathrm{R}$ and L.

It can be concluded from these experiments that only certain of the midline nuclei, viz., the nucleus reuniens and the rhomboid nucleus, project directly to the cortex on the medial surface of the hemisphere. That the intralaminar nuclei likewise have a precise topical projection to the head of the striatum is demonstrated by the experiments of the following group.

In Rabbit 44 an electrolytic lesion was placed in the dorso-medial quadrant of the cerebral hemisphere extending from immediately in front of the anterior horn of the lateral ventricle back to the ventral hippocampal commissure. It either directly involves or isolates a large part of the anterior limbic, cingulate, and precentral agranular areas. The caudate nucleus is damaged from its anterior end back to the level of the middle of the septum; anteriorly the dorsal half of the nucleus is involved but the extent of the damage gradually diminishes posteriorly until it is limited to the dorso-medial ventricular margin. The dorsal part of the lateral septal nucleus is destroyed throughout its anteroposterior extent. In the thalamus the most significant cellular degeneration is in the nucleus centralis lateralis and the adjacent part of the nucleus paracentralis; in these areas almost complete cell loss has occurred and the few remaining cells are shrunken and pyknotic (Plate 4). The nucleus centralis medialis shows shrinkage and pallor of the cells in its lateral part only, while the parafascicular, parataenial, and paraventricular nuclei show no change. The rhomboid nucleus and nucleus reuniens are severely degenerated. In the nucleus ventralis anterior and the rostral portion of the reticular nucleus there is a wedge-shaped area of cell loss and gliosis immediately ventral to the degenerate antero-medial and medial half of the antero-ventral nuclei. The other main nuclei 


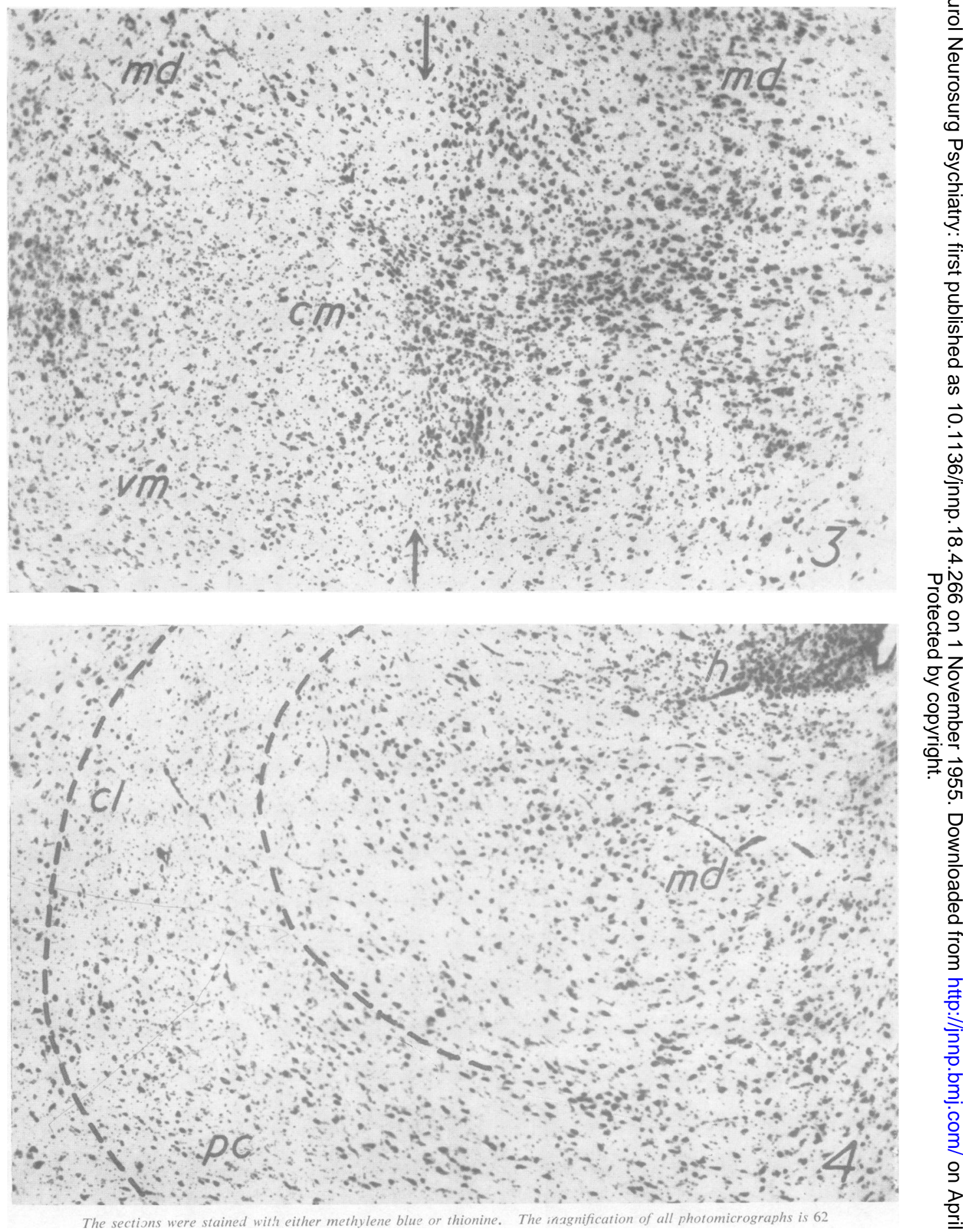

PI.ATE 3.-Degeneration in the nucleus centralis medialis and the adjacent medio-dorsal and ventro-medial nuclei in experiment $R$ 251L. I'I.ATE 4.-Degeneration in the nuclei centralis lateralis, paracentralis, and the adjacent part of the medio-dorsal nucleus in experiment $R 44$. 

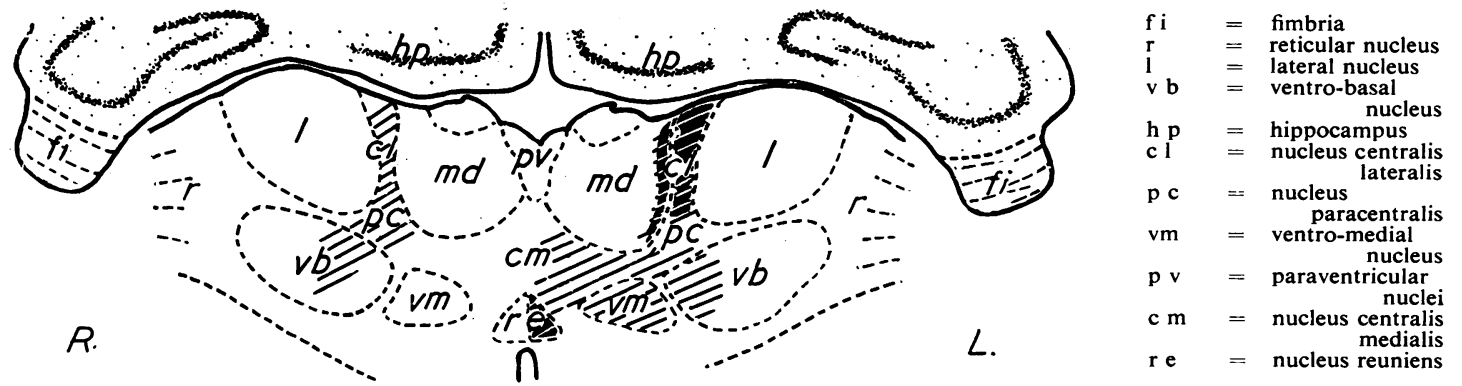

Fig. 7.-The thalamic degeneration in $\mathrm{R} 251$, most severe in the $\mathrm{nn}$. centralis lateralis and reuniens of the right side.

which show degeneration are the lateral part of the medio-dorsal (close to the degenerated nucleus centralis lateralis), the ventro-medial, and the medial parts of ventro-lateral and ventro-basal nuclei.

In $\mathrm{R} 251 \mathrm{~L}$ there is a sharply circumscribed electrolytic lesion in the dorsal one-third to one-half of the head of the caudate nucleus with only minimal involvement of the immediately adjacent cortex and subcortical white matter ; the damage does not extend behind the level of the genu of the corpus callosum (Fig. 6). The nuclei reuniens and rhomboideus are severely degenerate; in the nucleus centralis medialis there is a marked cell loss while the remaining cells are shrunken and compacted together and the nucleus paracentralis shows a slight diminution in the number of cells and many of the persisting cells are poorly stained (Plate 3). The nucleus centralis lateralis is almost completely atrophied with an accompanying severe gliosis; this degeneration is directly continuous with a similar small area in the lateral part of the medio-dorsal nucleus. The parataenial, parafascicular, and paraventricular nuclei show no change. The rostral end of the reticular nucleus and the overlying nucleus ventralis anterior show slight cell loss as well as compacting of the remaining cells beneath the partially degenerated antero-medial nucleus. The medial thirds of the ventro-lateral and ventro-basal nuclei together with the ventro-medial nucleus have undergone partial degeneration (Fig. 7).

In the opposite hemisphere of R 251 a lesion was placed in the dorsal half of the putamen from the level of the genu of the corpus callosum back to the level of crossing of the anterior commissure. Laterally the claustrum and the overlying neocortex have been involved while a narrow medial extension of the lesion crosses the upper end of the internal capsule to encroach upon the dorso-lateral margin of the head of the caudate nucleus (Fig. 6). In the thalamus of this side the cell degeneration is almost confined to the intralaminar nuclei and the only changes in the main nuclei are found in the medial part of the antero-ventral nucleus and in the middle thirds of the ventro-basal and ventro-lateral nuclei, all of which are partially degenerated. In the middle thirds of their medio-lateral extent the nucleus ventralis anterior and the adjoining reticular nucleus show slight cell loss and gliosis. Partial cell loss and gliosis have occurred in the nucleus centralis lateralis without any apparent change in the medio-dorsal nucleus while in the nucleus paracentralis there is a diffuse thinning out of the cells. There is a severe cell loss in the ventral half of the parafascicular nucleus, particularly in its posterior part in which the portion of the nucleus lateral to the habenulo-peduncular tract is almost completely atrophied (Plate 5). All the midline nuclei (parataenial, paraventricular, rhomboid, and reuniens) and the $n$. centralis medialis are unchanged (Fig. 7).

That the projection fibres of the midline and intralaminar nuclei neither terminate in nor traverse the medial preoptic area is indicated in experiment Rabbit 45. In this animal there is a well localized lesion in the medial preoptic area and the anterior hypothalamus. The electrode had been passed in a slightly caudal direction through the hemisphere close to the midline starting at the level of the anterior commissure ; the posterior two-thirds of this commissure is destroyed together with the dorsal third of the medial preoptic area as far laterally as the bed nucleus of the stria terminalis. Caudally the lesion reaches as far back as the paraventricular hypothalamic nucleus destroying the descending column of the fornix. Near its caudal limit the upper margin of the lesion abuts on the thalamic reticular nucleus while laterally it adjoins (but does not involve) the inferior thalamic radiation. There is no retrograde degeneration in any of the thalamic nuclei as a result of this lesion; the absence of degeneration in the anterior paraventricular nucleus in particular should be noted.

The complete lack of degeneration in the midline and intralaminar nuclei in the thalamus of the left 

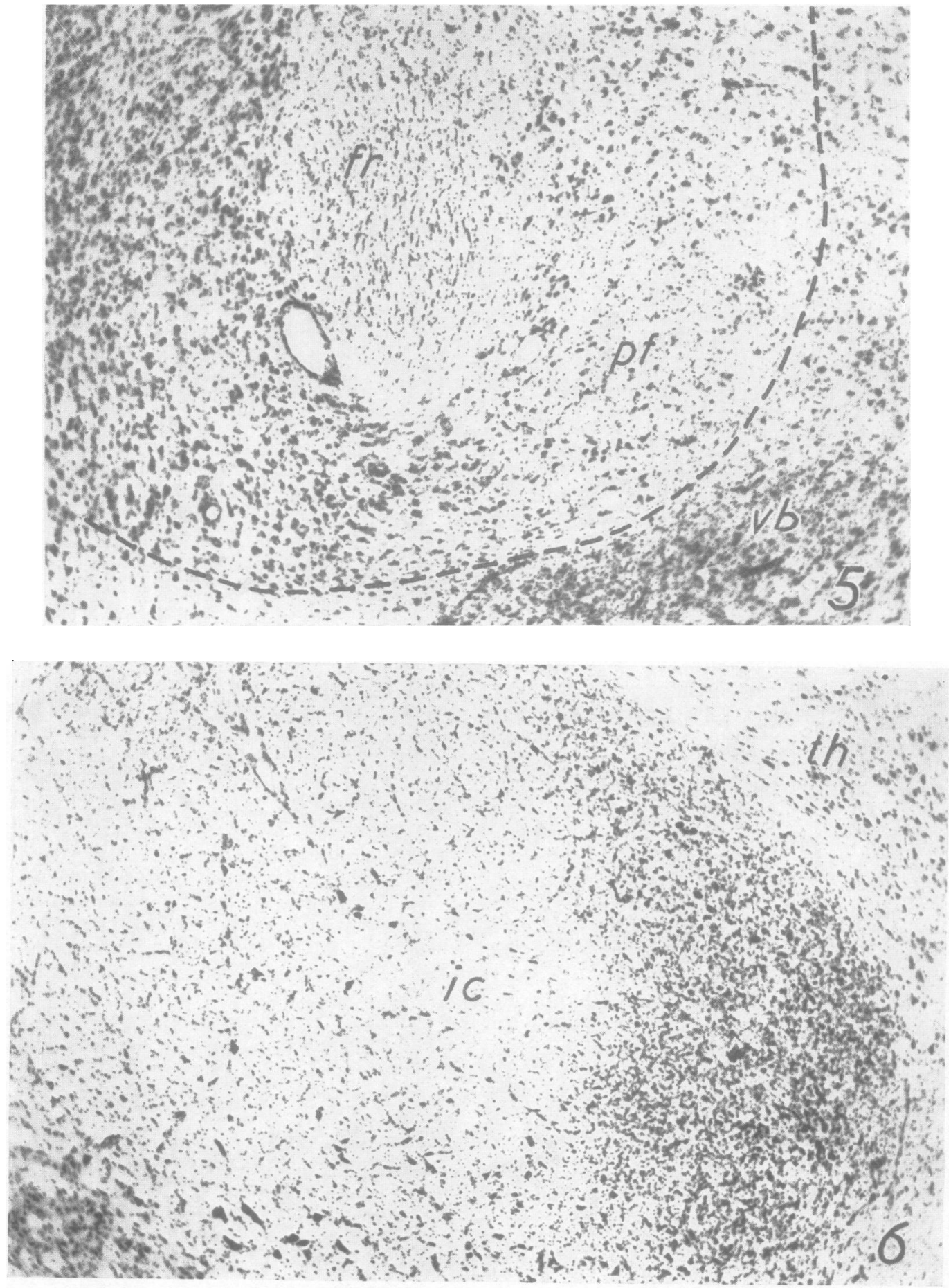

The sections were stained with either methylene blue or thionine. The magnification of all photomicrographs is 62.

Plate 5.-Degeneration in the lateral half of the parafascicular nucleus in experiment $\mathbf{R} 251 \mathbf{R}$

Plate 6. - The severe gliosis in the ventral third of the internal capsule following a large lesion in the corpus striatum in experiment $R 42$. 
hemisphere in Rabbit 35 excludes a projection from these nuclei to the septum. On this side the knife has entered the dorsal margin of the septum from the opposite limbic cortex at the level of the anterior end of the medial septal nucleus and extends back to the ventral hippocampal commissure. The entire medial septal nucleus, the posteromedial half of the lateral septal nucleus, and the vertical limb of the diagonal band nucleus have been directly damaged, while the rest of the lateral septal nucleus shows severe cell loss with shrinkage and compacting of the remaining cells.

The final experiments to be described demonstrate the pathway of the efferent fibres from the midline and intralaminar nuclei. In R 52 a lesion was placed in the middle third of the internal capsule extending forwards from the anterior end of the thalamus into the dorsal part of the striatum ; the most medial fibres of the internal capsule, however, have been spared. Although the midline nuclei, i.e., the reuniens, rhomboid, and parataenial, are unchanged, all the intralaminar nuclei, including the parafascicular, are totally degenerated, showing that there is a precise and independent pathway for these two groups of nuclei. Selective degeneration has occurred in the nucleus reuniens and rhomboid nucleus in R 57 following a small lesion in the most medial portion of the internal capsule at the level of the rostral end of the striatum, which is also damaged. This experiment when taken in conjunction with the earlier findings suggests that the fibres from these nuclei, after leaving the internal capsule, pass round the rostral end of the head of the caudate nucleus and the anterior limit of the lateral ventricle to reach the medial surface of the hemisphere. That this is indeed the case is seen in $R$ 24R in which gliosis is present in the white matter medial to the anterior horn of the lateral ventricle well in front of the cortical lesion.

\section{Discussion}

The conclusion reached by Rose and Woolsey (1943) that the nuclei of the midline and internal medullary lamina are telencephalic dependencies has been fully substantiated by the experiments described in this and in our previous study (Powell and Cowan, 1954). However, with the exception of the recent work of Fortuyn (1950), and Stefens and Fortuyn (1953), no attempt has been made to define the projection of the individual elements of this system. An analysis of the material presented here in conjunction with earlier observations has to some extent clarified this problem.

That the nucleus reuniens and the rhomboid nucleus (as defined above) have a projection which is independent of the remainder of the midline and intralaminar nuclei has been clearly shown. In the experiments of the third group, these nuclei underwent complete selective, retrograde cell degeneration following lesions confined to the cortex on the medial surface of the hemisphere without any involvement of subcortical tissue. A correlation of the extent of cortical damage with the severity of degeneration in these experiments indicates that they project to the region of the infralimbic cortex (area 25 of Brodmann). This was conclusively established in the critical experiment $R 24 \mathrm{~L}$ in which the cortical damage was confined to the infralimbic area and the ensuing retrograde degeneration to the nucleus reuniens and the rhomboid nucleus. In their study of the connexions of the anterior nuclei in the rabbit and cat, Rose and Woolsey (1948) mention that in one experiment (RW18) severe degeneration was present in their "medio-ventral nucleus" ( $=$ nucleus reuniens) after a lesion confined largely to the infralimbic cortex and conclude from this and their other experiments, in which this region was incidentally involved, that this cortical area represents the projection field of the nucleus. A similar conclusion was reached by Fortuyn (1950) in his study of the paramedian and submedial nuclei.

The experiments of the third group also make it clear that no other midline thalamic nuclei project to the medial surface of the hemisphere or the septum. It has been suggested by some (Stoffels, 1939; Lashley, 1941 ; Fortuyn, 1950) that the parataenial nucleus may project to the most basal part of the medial surface of the hemisphere (retrobulbar area and taenia tecta), but this conclusion is not supported by our experiments. That the nucleus does project to the medial part of the rostral telencephalon is apparent from the consistent retrograde degeneration which occurs in the experiments with large lesions in this area (e.g., Rabbits 2 and 15). Degeneration was also seen in this nucleus in experiment $R$ 55R in which the medial parts of the caudate nucleus and nucleus accumbens were damaged together with the cortex on the medial surface of the hemisphere. However, complete destruction of the head of the caudate nucleus with only marginal involvement of the nucleus accumbens (e.g., Rabbits 60 and 59) results in no marked retrograde change in the parataenial nucleus. From this it must be concluded that the parataenial nucleus is connected either with the nucleus accumbens or with the overlying olfactory tubercle. In our previous study (Powell and Cowan, 1954) a projection of the parataenial nucleus to the rostromedial two-thirds of the olfactory tubercle was 
excluded by experiment R.12. This experiment has been re-examined and it has been confirmed that the lesion is strictly confined to this portion of the olfactory tubercle with little or no involvement of the nucleus accumbens and that there is no apparent change in the parataenial nucleus. It would thus appear that the efferent fibres of the parataenial nucleus terminate either in the nucleus accumbens or in the lateral part of the olfactory tubercle. In some of our experiments in which the nucleus accumbens was marginally involved there was often a suggestion of cell loss along the margins of the parataenial nucleus although no serious atrophy of the nucleus was apparent. Since we have no lesion which completely destroys the nucleus accumbens without involvement of other adjacent structures this point has not been conclusively determined. Our findings are not entirely at variance with the observations of the authors quoted above, as it is clear from the diagrams of their lesions that the nucleus accumbens or the adjacent internal capsule were usually involved (cf. Fortuyn, 1950). Furthermore, as Rose and Woolsey (1943) point out, Stoffels' (1939) suggestion that the taenia tecta is the projection area for this nucleus seems to have been an indirect conclusion only.

In our previous study (Powell and Cowan, 1954) degeneration of the anterior and posterior paraventricular nuclei was described in six experiments in some of which an extensive lesion of the basal forebrain areas extended back into the anterior hypothalamus; the only other description of degeneration in these nuclei is that of Walker (1936) in the monkey after a lesion in the medial preoptic and anterior hypothalamic areas. In the first two experiments described in the present communication, partial degeneration has occurred in these nuclei after large lesions of the striatum and basal forebrain areas but without involvement of the hypothalamus. Further, in one experiment (Rabbit 45) in which the lesion was confined to the medial preoptic and anterior hypothalamic areas no degeneration was seen in these nuclei. It thus appears that these nuclei will undergo degeneration following extrathalamic lesions but it has not been possible to determine their projection (cf. Rose and Woolsey, 1943). The nucleus centralis medialis, on the other hand, appears to project in close association with the intralaminar nuclei-paracentralis and centralis lateralis - to the caudate nucleus. In the majority of the experiments, in which the lesions were confined largely to the dorso-lateral part of the head of the caudate nucleus, the severity of the thalamic degeneration was most marked in the nucleus centralis lateralis and was usually least marked in the nucleus centralis medialis. Although it has not been possible to determine the precise termination of the efferent fibres of each of these elements, in experiments involving different parts of the head of the caudate nucleus the distribution and severity of the retrograde degeneration in these thalamic nuclei varied quite definitely, suggesting that each has an independent projection to the caudate. That the projection of these three intralaminar nuclei is also independent of the principal thalamic nuclei is apparent from those experiments in which they have undergone degeneration without any appreciable change in the adjacent main nuclei. This conclusion is in accord with the findings of Stefens and Fortuyn (1953), and it is now well established that there is a direct thalamo-caudate projection.

Although it has been frequently suggested that the parafascicular nucleus of lower mammals projects to the striatum (cf. Stefens and Fortuyn, 1953), the only experimental evidence which has been adduced to support this hypothesis is that of Gerebtzoff (1940). The experiments described in our paper indicate quite clearly that the parafascicular nucleus projects to the putamen, and, if the lateral part of this nucleus is indeed homologous with the centre median of primates (Le Gros Clark,, $\mathbb{D}$ 1931), it too would have a similar projection. Thiso is in accord with the reports of degeneration in the centre median in human pathological material with lesions involving the striatum (Vogt and Vogt, 1941 ; McLardy, 1948).

Our findings on the degeneration in the reticular nucleus are similar to those previously described (Rose, 1952 ; Chow, 1952 ; Powell and Cowan, 1954). The nucleus ventralis anterior, which has recently been shown to be an important element in the diffuse projection system of the thalamus (Starzl and Magoun, 1951 ; Hanbery and Jasper, 1953), frequently showed marked degenerative changes in these experiments. These findings will, however, not be discussed here as the efferent connexions of this nucleus are being further investigated.

From the variety of lesions which have been placed, and by tracing out the ensuing gliosis, it has been possible to determine the precise pathway of the efferents from the midline and intralaminar nuclei to the striatum and the infralimbic area. The fibres leave the thalamus in the inferior thalamic radiation, pass forwards in the ventral third of the internal capsule, the fibres from the parafascicular nucleus diverging laterally into the putamen while the remainder continue forwards towards the head of the caudate nucleus. Here the fibres from the 
nucleus reuniens, the rhomboid, and the parataenial nuclei occupy the most ventral part of the internal capsule, while the intralaminar projection fibres which lie more dorsally swing medially into the caudate nucleus. The fibres to the infralimbic cortex then course around the rostral end of the caudate nucleus before terminating in the medial surface of the hemisphere.

The observations reported herein provide an anatomical basis for the physiological work of Stoupel and Terzuolo (1954) and Shimamoto and Verzeano (1954) on the relation of the caudate nucleus to the diffuse thalamic projection system.

\section{Summary}

The efferent connexions of the midline and intralaminar nuclei of the thalamus have been investigated in the rabbit by the method of retrograde cell degeneration.

It has been established that these nuclei have extrathalamic connexions; with the exception of the paraventricular nuclei, they project by way of the inferior thalamic radiation to the corpus striatum and the adjacent cortex of the medial surface of the hemisphere.

The intralaminar nuclei, centralis medialis, paracentralis, and centralis lateralis, project to the head of the caudate nucleus while the parafascicular nucleus is connected with the putamen.
The midline nuclei reuniens and rhomboideus project directly to the cortex of the infralimbic area.

The precise connexions of the cells of the paraventricular and parataenial nuclei could not be determined but the latter is probably connected with the nucleus accumbens.

We wish to thank Mr. Michael Lindsey and Mr. Brian Purvis for valuable technical assistance.

\section{REFERENCES}

Chow, K. L. (1952). J. comp. Neurol., 97, 37

Council for International Organizations of Medical Sciences (1954). Brain Mechanisms and Consciousness : A Symposium. Black-

well, Oxford.
Cowan, W. M., and Powell, T. P. S. (1954). Proc. roy. Soc. B., 143, 114.

Fortuyn, J. Droogleever (1950). Folia psychiat., Amst., 53, 213.

Gerebtzoff, M. A. (1940). J. Belge de Neurologie et de Psychiatrie, 40, 407.

Gurdjian, E. S. (1927). J. comp. Neurol, 43, 1

Hanbery, J., and Jasper, H. (1953). J. Neurophysiol., 16, 252.

Lashley, K.S. (1941). J. comp. Neurol., 75, 67.

Le Gros Clark, W. E. (1931). Quoted by Rioch, D. M. J. Anat. Lond., 65, 324.

McLardy, T. (1948). Brain, 71, 290.

Powell, T. P. S., and Cowan, W. M. (1954). J. Anat., Lond., 88, 307.

Rose, J. E. (1952). Res. Publ. Ass. nerv. ment. Dis., 30,454

Rose, and Mountcastle, V. B. (1952). J. comp. Neurol., 97, 441.

二, and Woolsey, C. N. (1943). Bull. Johns. Hopk. Hosp., 73, 65.

-, (1948). J. comp. Neurol., 89, 279.

Shimamoto, T., and Verzeano, M. (1954). J. Neurophysiol., 17, 278. Starzl, T. E., and Magoun, H. W. (1951). Ibid., 14, 133.

Stefens, R., and Fortuyn, J. Droogleever (1953). Schweiz. Arch. Neurol. Psychiat., 72, 299.

Stoffels, J. (1939). Mém. Acad. roy. Méd., Belg., 2 ser. 1, no. 2, p. 1. Stoupel, N., and Terzuolo, C. (1954). Acta neurol. psychiat. belg., $54,239$.

Vogt, C., and Vogt, O. (1941). J. Psychol. Neurol., 50, 32.

Walker, A. E. (1936). J. comp. Neurol., 64, 1.

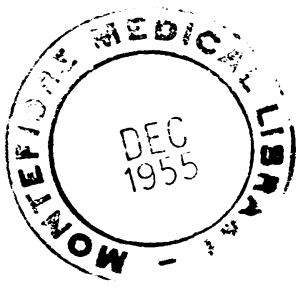

